\title{
Implementación de controles tecnológicos para la Mejora Continua en la reducción de accidentes en la construcción de piques
}

\author{
Implementation of technological controls for Continuous \\ Improvement in reducing accidents in shaft construction
}

\author{
Kelvis Berrocal Argumedo ${ }^{l}$ \\ ${ }^{1}$ Universidad Nacional de San Cristóbal de Huamanga, Perú.
}

\begin{abstract}
RESUMEN
El presente estudio tiene como objetivo general determinar el efecto de la implementación de los controles tecnológicos para la Mejora Continua en la reducción de accidentes en la construcción de piques - Corporación Minera Géminis SAC.

El estudio se basa en el análisis estadístico de los índices de accidentabilidad y las medidas de control: geotécnicas, administrativas y de seguridad propiamente dicha, así como la participación responsable, comprometida y apropiada en el desarrollo y optimización de la seguridad mediante el control, principalmente tecnológico para reducir los accidentes, como se ha comprobado entre los años 2010 al 2017.

Están definidas las variables del estudio y son analizadas de forma exhaustiva para determinar la relación que existe desde una perspectiva principalmente cuantitativa que permite una mejor apreciación de los efectos.

Finalmente, se consideran conclusiones que manifiestan la intención de la investigación, y se proponen algunas sugerencias para el mejor control de la seguridad.
\end{abstract}

Palabras clave: control tecnológico, accidente laboral, minería de piques, seguridad laboral.

\section{AbSTRACT}

The general objective of this study is to determine the effect of the implementation of technological controls for Continuous Improvement in reducing accidents in shaft construction - Geminis Mining Corporation, Peru.

The study is based on the statistical analysis of accident rates and control measures: geotechnical, administrative and security itself, as well as responsible, committed and appropriate participation in the development and optimization of security through control, mainly technological, to reduce accidents, as has been proven from 2010 to 2017.

The study variables are defined and exhaustively analyzed to determine the relationship that exists from a mainly quantitative perspective that allows a better appreciation of the effects.

Finally, conclusions are considered that express the intention of the investigation, and some suggestions are proposed for the better control of security.

Keywords: technological control, occupational accident, shaft mining, occupational safety. 
ISSNe: 2617-9156

\section{INTRODUCCIÓN}

$\mathrm{E}$ presente artículo hace un análisis de la salud y la seguridad de los trabajadores como la acción fundamental de las empresas mineras y el Estado, que implica mejorarlas para controlar la accidentabilidad o índices de accidentes, obviar las pérdidas de tiempo e incrementar la productividad, reduciendo pérdidas de capital y controlando los riesgos de conflictos laborales, sociales o ambientales, con la finalidad de garantizar la integridad física y mental de los trabajadores y la seguridad en las minas, debiendo implementar acciones permanentes y bajos índices de siniestralidad.

La seguridad y la salud adecuadas en el trabajo constituyen una necesidad que implica eficiencia al salvaguardar la integridad de las personas, lo que permite la satisfactoria productividad, teniendo como necesidades la implementación de los controles tecnológicos y su cumplimiento estricto para posibilitar la sustentabilidad de la empresa a corto, mediano y largo plazo.

Perú tiene una riqueza de minerales distribuidos en la costa, sierra y selva. Estos recursos vienen siendo explotados de diversas maneras, desde la minería informal hasta una formal mecanizada o convencional, razón por la cual se considera como la actividad más importante en la economía peruana porque genera valor agregado y aporta $10 \%$ al producto bruto interno (PBI), mayores divisas e ingresos fiscales por impuestos, la creación de empleos directos e indirectos, y la mejora del crecimiento potencial de la actividad económica. Además, atrae inversiones, nacionales y extranjeras. (Montero, 2018, p.10).

A nivel mundial los accidentes en las diversas industrias son preocupantes como afirma, el director de la OIT con sede en Argentina, Pedro Furtado de Oliveira, revelando que: "En la actualidad mueren 7.600 personas por día como consecuencia de accidentes de trabajo o enfermedades profesionales y una herramienta imprescindible son las políticas preventivas que permitirían evitar muertes y enfermedades profesionales" (Furtado de Oliveira, 1, 2019)

En el Perú las estadísticas en materia de salud y seguridad revelan algunas verdades amargas, pues según el estudio realizado por el Ministerio de Trabajo y Promoción del Empleo, se producen más de 20 mil accidentes de trabajo cada año, estas cifras en el año 2018 fue de 20,136 colaboradores en las siguientes industrias:

\section{Figura 1:}

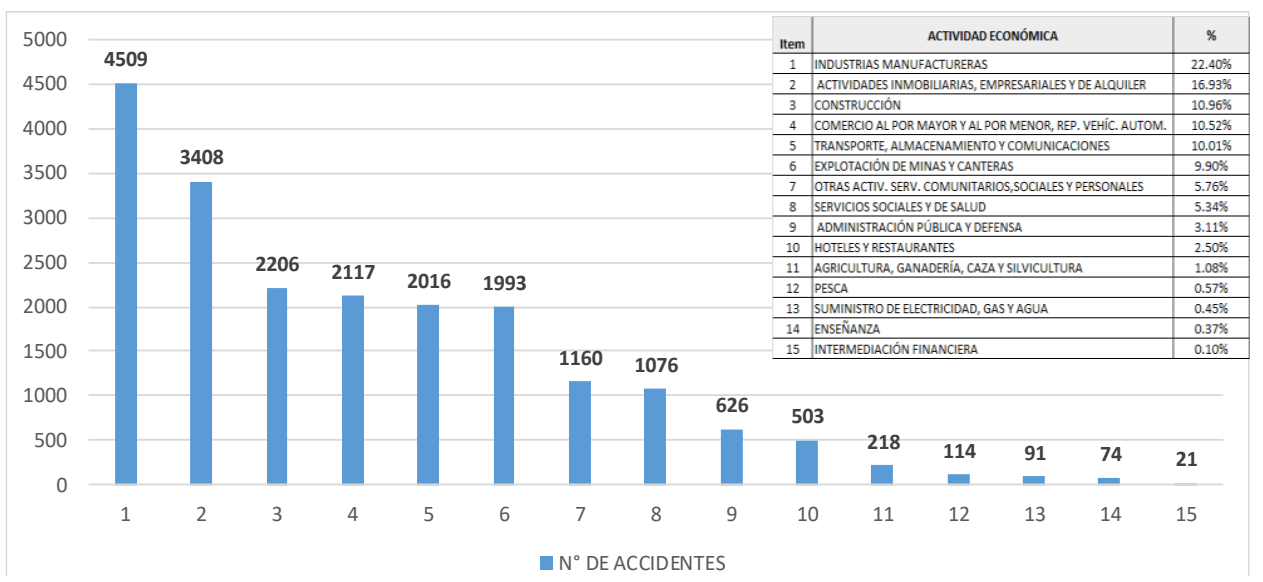

Accidentes notificados según actividad económica año 2018.

Fuente: Elaboración propia.

Por otro lado, las estadísticas de los accidentes mortales suman un total de 150 para el año 2018 distribuidos en cada sector de la industria:

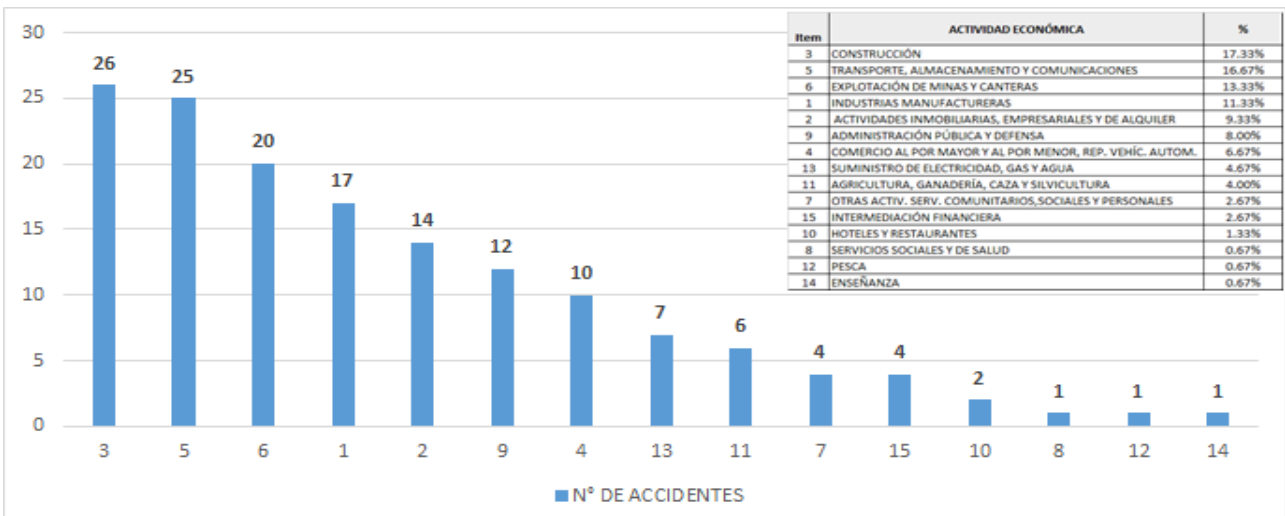

Figura 2:

Accidentes Mortales Según Actividad Económica 2018.

Fuente: Elaboración propia. 
En nuestra patria, no obstante que el Estado a través de los diferentes órganos gubernamentales dictan normas referentes a la seguridad y salud en el trabajo, y suman esfuerzos además de las iniciativas propias de las empresas privadas implementando diversos sistemas de gestión de seguridad con la finalidad de proteger el recurso más valioso de las empresas que son los colaboradores, sigue siendo un tema muy importante por la ocurrencia de accidentes que en muchos casos tiene las mismas causas (Figura 3).

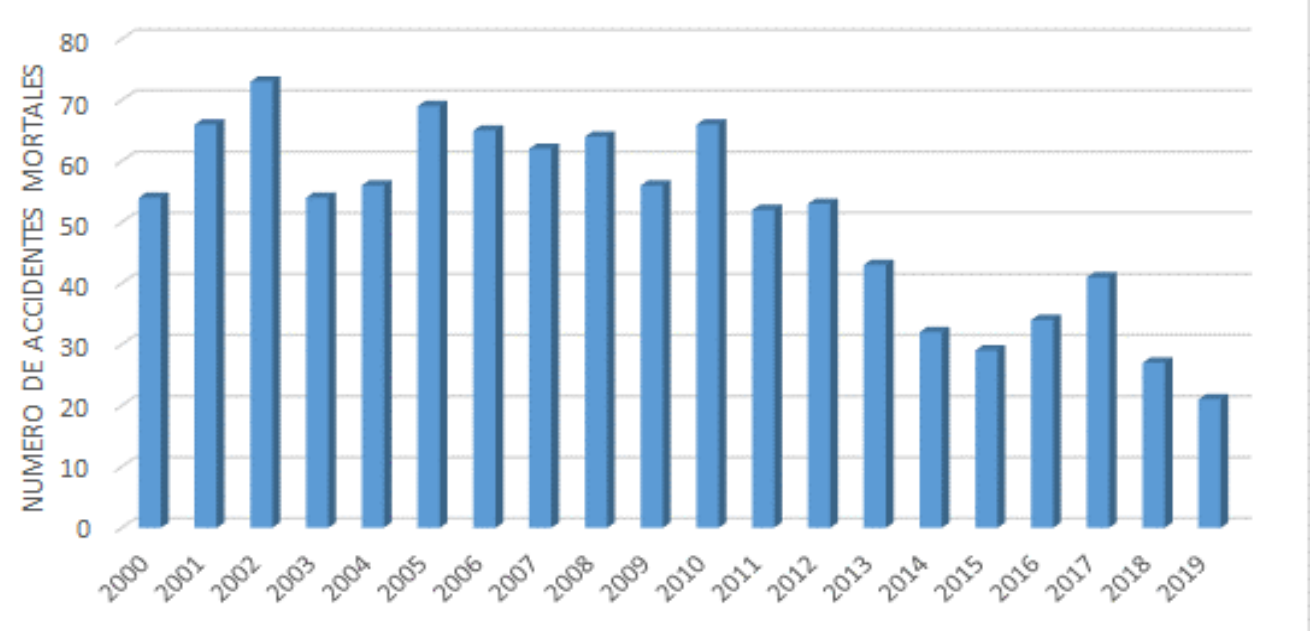

Figura 3:

Accidentes mortales del año 2000 al 2019.

Fuente: Elaboración propia.

En la actualidad, la estadística nacional en la industria minera publicada por el Ministerio de Energía y Minas nos muestra una información del 2000 hasta el 2019, y de acuerdo a la Figura $\mathrm{N}^{\circ} 03$ la pendiente de los accidentes mortales es muy fluctuante, esto debido a diversos factores y tipos de laboreo que se viene realizando, como recuperación de pilares, escudos, puentes, y si se desarrollan labores de alto riesgo como los piques.

Las labores verticales como los piques "A pesar de que es una de las actividades mineras más riesgosas, es posible desarrollarla con un buen respaldo de ingeniería y control que nos permita transformarla en una operación segura" (Salinas, 1998, p55).

En la industria minera los piques tiene la función de izaje de personal, requiere equipos, materiales, y es considerado dentro de las operaciones unitarias "La actividad más importante en una operación minera, pues es la extracción del mineral (limpieza), la que se puede realizar por movimiento en forma horizontal (locomotora, scoop, volquetes), en forma vertical (izaje), por inclinados, rampas y zigzag" (De la Cruz, 2000, p. 01).

Por ello es más frecuente que las empresas mineras migren a un sistema de izaje mediante los piques; “...es necesario conocer la naturaleza del trabajo, y de todos los factores que influyen en desarrollar este tipo de labores, conocer los elementos relacionados con este tipo de trabajo como maquinarias, equipos, herramientas, condiciones de temperatura, geomecánica, ventilación, ritmos de trabajo, que puedan influir negativamente a la salud del trabajador" (Bavaresco, 2009, p. 5).

Dentro de los riesgos principales inherentes al proceso constructivo del pique están:

- caída de personal a distinto nivel
- desprendimiento de rocas

- atrapamiento o aprisionamiento

- manipulación de herramientas

- explosivos

- manipulación de materiales

- falta de ventilación

- incumplimiento a procedimientos

- electrocuciones o descargas de energía eléctrica, entre otras.

El MINEM clasifica a los incidentes en 68 ítems, y desde el año 2006 al 2015 las estadísticas nos muestran situaciones como 1) Desprendimiento de rocas: $7.59 \%$, 4) Falta de Ventilación: $6.12 \%, 5)$ Incumplimiento a procedimiento: $5.48 \%, 6$ ) Herramientas: $5.05 \%$ y 9) Energía eléctrica: $3.87 \%$, que sin duda son incidentes propios al proceso constructivo de piques, y que si no son controlados traen como consecuencias accidentes leves, incapacitantes o mortales, afectando a las familias que quedan desamparadas, y hechos adicionales como contaminación ambiental, conflictos comunales, imagen empresarial deteriorada, problemas económicos y financieros, multas legales, paralizaciones de las operaciones y de la mina misma.

Dentro del análisis de los informes de investigación de los accidentes las causas se han resumido en: Factor humano, Falta de planeamiento operacional, Deficiencia en los estándares operativos, Pluriculturalidad, Débil interacción y débil liderazgo del supervisor y Compromiso de gerencia no visible.

La presente investigación se limita a estudiar y prevenir los accidentes en la construcción de piques a través de la pregunta: ¿de qué manera la construcción de piques están innovando su tecnología y mejora continua en sus procesos para evitar y prevenir accidentes?

El presente estudio se justifica porque en la construcción y 
ISSNe: 2617-9156

mantenimiento del pique, se presenta riesgos que actualmente son los causantes de accidentes mortales en la industria minera, debido a los controles deficientes e inadecuados implementados por las empresas mineras.

Esta investigación fortalece la gestión de seguridad de las empresas que desarrollan construcciones de piques que son considerados labores de alto riesgos en el sector minero, pues permite proporcionar acciones, controles, procedimientos y estándares en el proceso constructivo, que sin duda son una herramienta de gestión y control en la prevención de los accidentes.

Sirve de respaldo a las empresas mineras, directivos de las empresas, ingenieros, supervisores que ante estos eventos son procesados en forma penal y civil, asimismo evitará que las empresas y contratistas mineras sean sancionadas económicamente por las entidades del Estado.

El control tecnológico de seguridad, según Chiavenato (2001), precisa que "La tecnología incluye aspectos físicos y concretos (hardware), como máquinas, instalaciones, equipos, circuitos, etc., así como aspectos conceptuales y abstractos (software), como política, directrices, procesos, procedimientos, normas y reglamentos, rutinas, planes, programas y métodos de trabajo" (Chiavenato, 2001, p. 97).

De acuerdo a Herrera (2013) se refiere: "Como la aplicación con fines prácticos de los conocimientos científicos y que se traduce en el reemplazo de los distintos tipos de trabajo por modernas máquinas, procedimientos y técnicas de producción más eficientes, creación de nuevos productos, y cambios no sólo en la producción, sino también en los métodos de distribución. Pero las tecnologías a menudo se basan en conocimientos no científicos ni rigurosos, en experiencias más o menos elaboradas que surgen de la misma práctica" (Herrera, 2013, p. 59).

Sus aplicaciones están orientadas a mejorar la Prevención de Riesgos Laborales desempeñando distintas funciones:

a) Operación manual

b) Operación autónoma

c) Tele operación

d) Dispositivos móviles

e) Drones

f) Plataformas digitales

g) Simuladores

h) Big Data

\section{Control tecnológico en la seguridad}

En toda actividad laboral son esenciales la seguridad y la salud, siendo prioritarios: el monitoreo, la capacitación o manejo de información, para mejorar la seguridad en el trabajo, considerando la comunicación eficiente, rápida y menos complicada, y es esencial para proteger el entorno laboral de accidentes y responder eficazmente a los sucesos adversos.

El control tecnológico posibilita que los trabajadores realicen actividades en todo terreno cuando los medios con los que se realizan han sido controlados, estas situaciones de riesgo no solo están identificadas o evaluadas, sino que son reconocidas por los trabajadores quienes se desempeñan favorablemente, porque han:

- Agilizado la comunicación

Attribution-NonCommercial 4.0 International (CC BY-NC 4.0)
- Fortalecido las capacidades del personal; y

- Mejorado el monitoreo

Es importante resaltar que todo depende de acciones de respuesta ágiles y de determinaciones adecuadas o pertinentes, las que se convierten en elementales para reducir riesgos de accidentes, lesiones y muertes.

Es necesario precisar que se tiene varias tecnologías innovadoras que en el tiempo cada vez permiten que los entornos de trabajo sean más seguros para los colaboradores de una empresa. Gracias al avance de la tecnología, cada vez son más los efectos favorables en seguridad y salud laboral. Las empresas están utilizando la tecnología digital y software para ayudar a sus empleados a ser más conscientes de su entorno de trabajo y los riesgos y peligros que pueden enfrentar, como son:

\section{Software para capacitación}

Un software que genera sesiones de entrenamiento para los empleados en un entorno determinado, que recrea sitios de trabajo y las condiciones de trabajo para permitir a los empleados conocer los peligros y riesgos potenciales antes de que entren en los lugares de trabajo reales.

La tecnología para la seguridad en el trabajo también está mejorando las comunicaciones gracias al desarrollo de tecnología que puede rastrear datos biométricos, habilitar llamadas de video y voz, escanear códigos de barras y detectar la exposición a elementos o situaciones dañinas.

\section{Accidentes en la construcción de piques}

Todo evento repentino que sobrevenga en el trabajo y produzca en el trabajador una lesión, perturbación funcional, invalidez o muerte. Se considera accidente laboral cuando ocurre en la ejecución de órdenes del empleador, o durante la ejecución de una actividad bajo su responsabilidad, así ocurra fuera del centro de trabajo y de las horas de trabajo.

\section{La extracción por pique vertical}

Es una excavación vertical de arriba hacia abajo, de manera circular, cuadrada o rectangular y cuya variada dimensión dependerá de los fines de trabajo para lo cual es propuesta dicha construcción; siendo los casos, el transporte de equipo y personal, los servicios (materiales) y la extracción de mineral y desmonte de las zonas bajas. Su aplicabilidad es circunscrita a yacimientos de gran profundidad y donde la accesibilidad por superficie al punto de llegada donde se ubica éste, es bastante lejos.

\section{Sus ventajas son:}

- Permite el acceso de los operarios en las zonas más profundas de un modo más rentable y rápido, extrayendo el mineral por izado de skip, hasta niveles superiores.

- Bajo costo de operación, en lo referente al izaje de mineral o desmonte;

- Es una tecnología limpia, que no produce contaminación en la zona de trabajo

- Menor distancia de excavación para alcanzar la mayor profundización.

Y sus desventajas son:

- Mayor tiempo de excavación para una determinada profundización.

https://doi.org/10.46908/tayacaja.v4i2.180 
ISSNe: 2617-9156

- Centralización del izaje de mineral o desmonte, de los servicios y del personal como la accesibilidad de los equipos.

- Interferencia de las operaciones, cuando los requerimientos de transporte, movimientos de equipos y personal son efectuados de manera simultánea por el pique.

- Limita el acceso de equipos con dimensiones mayores a la infraestructura realizada del pique.

- El rango de izaje máximo, tanto de mineral como de desmonte, es básicamente limitado a la capacidad del winche de izaje sin perspectiva de crecimiento de la producción.

- Requiere de personal en cuadrillas especializadas de mantenimiento.

- En caso de siniestros, su flexibilidad no concuerda con la celeridad de evacuación de los equipos y personal.

\section{MARCO METODOLóGico}

Es un trabajo de investigación que utiliza el modelo de enfoque dominante aplicado, debido a que el conocimiento derivado se propondrá políticas y programas de acción correctiva del fenómeno en estudio.

De acuerdo con Hernández, Fernández y Baptista (2014), el método que corresponde a la presente investigación es el método inductivo en tanto "El objetivo está en ir de lo particular y concreto, a lo general y abstracto" (p. 8).

El diseño es correlacional para determinar la relación entre el uso del control tecnológico y la reducción de accidentes.

Se ha demostrado que los controles tecnológicos influyen en la reducción de accidentes en construcción de piques Corporación Minera Géminis SAC, al apreciar que la innovación de equipos influye en la reducción de accidentes en la construcción de piques de la CM Géminis SAC., la implementación de programas de geomecánica influye en la reducción de accidentes en la construcción de piques de la CM Géminis SAC y la innovación e implementación de nuevas herramientas de gestión influye en la reducción de accidentes en la construcción de piques de la CM Géminis SAC.

El estudio se ha aplicado a 45 trabajadores de campo que laboran en la Corporación minera Géminis SAC, seleccionados de forma arbitraria y circunstancial.

Para el estudio se han aplicado las siguientes técnicas de recolección:

A. Revisión documental y análisis, con los siguientes instrumentos:

- Levantamiento de información de accidentes y incidentes de seguridad, informes de investigación de accidentes

- Documentos del sistema de gestión de seguridad e índices de seguridad

- Diagnóstico o línea de base inicial del sistema de gestión en seguridad y salud en el trabajo, de acuerdo al anexo de la $\mathrm{RM} \mathrm{N}^{\circ}$ 050-2013-TR.

- Planes de minado y estudios de las condiciones geomecánicas.
- Información bibliográfica usando como fuente la relacionada a las estadísticas de accidentes mortales del Ministerio de Energía y Minas, OSINERMING, libros especializados en geomecánica, laboreo minero, seguridad y salud ocupacional en minería, normas, reglamentos, revistas, folletos e información vía Internet.

B. Observación directa - presencial. Se emplearon los siguientes instrumentos:

- Recopilación de reportes de actos y condiciones o libreta de Identificación de Peligros, cuaderno de seguridad

- Recopilación de los IPERC continuos de cada labor

- Reportes de ideas de Mejora Continua

- Reportes de evaluación y planos geomecánicos

- Reportes de las observaciones planeadas de trabajo

\section{RESUltado}

La industria de la minería subterránea es dinámica y evoluciona a través de tiempo, debido al uso de la tecnología en cada operación unitaria. El objetivo de la presente investigación es mejorar la gestión en prevención de riesgos en la ejecución de proyectos pique.

\section{Identificación de Riesgos Críticos en la Construcción del Pique}

Los riesgos críticos determinaron la peligrosidad de los riesgos y como éstos influyen en la ocurrencia del accidente o incidente de trabajo. Esta identificación se logra mediante los colaboradores, expertos en temas de seguridad en piques, estadísticas de accidentes e incidentes y de acuerdo a la magnitud de perdida producida por cada uno de ellos.

La identificación de los riegos críticos en el proceso constructivo de un pique permite tener una mejor apreciación de consecuencias de los riesgos y, a partir de estos datos, tomar todas las medidas de control respectivas. A continuación, resumimos las tareas críticas en la ejecución de piques:

Tabla 1.

Riesgos Críticos en la Construcción de Piques.

\begin{tabular}{cl}
\hline Ít. & Riesgos \\
\hline 1 & caída de rocas \\
2 & golpeado por caída de objetos \\
3 & caída de personal a distinto nivel \\
4 & electrocución \\
5 & intoxicación por inhalación de gases \\
6 & explosión \\
7 & atrapamiento por equipo en movimiento \\
\hline
\end{tabular}

Fuente: Elaboración propia.

\section{Implementación del control tecnológico \\ Evaluación geomecánica diaria}

Diariamente se realiza una evaluación para determinar las condiciones del macizo y a partir de ello se asumen medidas para la mejorar el sostenimiento y estabilización del macizo 
rocoso, aplicando la tabla GSI (Geological Strength Index, Índice de Resistencia Geológica) y de esta forma permite dar soporte a la labor de trabajo y de seguridad, determinando el tipo de sostenimiento. Este reporte o data es subido al sistema digital, permitiendo tener en tiempo real la información exacta de las recomendaciones del caso por el área de Geomecánica para la guardia. Fue favorable su uso ya que permitió asumir medidas de control que garantizan la integridad del macizo.

\section{Puntos de convergencia}

Díaz y De la Sota, (2005) mencionan que el control adicional para identificar y cuantificar las deformaciones del macizo rocoso es mediante el monitoreo de convergencias y mediante un procesamiento de datos y análisis estadístico. Los criterios de ubicación según estos puntos son:

- aspecto estructural

- intersecciones con galerías y cruceros

- calidad de roca

- hidrología

- alteración

- litología

- intersecciones con cruceros y chimeneas

- grado de fractura

- diseño de puentes de explotación

A partir de este monitoreo se realizó un plan de contingencia en función a la magnitud de convergencias; si la magnitud es alta, se debe tomar acciones inmediatas de replanteo o sostenimiento adicional. Otra técnica es realizar taladrados de alivio; el diámetro y la longitud están en función al estudio de los estallidos de roca de la mina. Otra alternativa es la revaluación del método de minado actual.

\section{Controles de ingeniería}

Se desarrollaron acciones directas de control técnico a partir de la ingeniería, como se explican a continuación:

a) Implementación de Controles de caída a distinto nivel

- Parrillas móviles metálicas para chimeneas.

- Plataforma metálica de servicio pique para instalación de guías y mantenimiento.

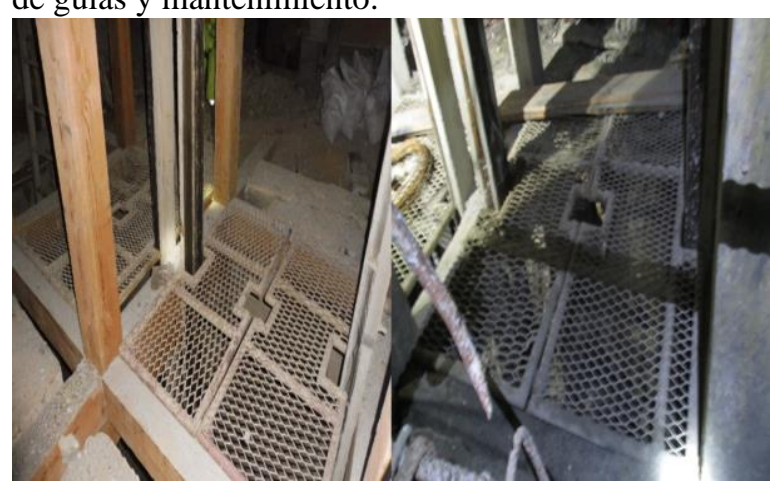

Figura 4:

Plataformas Metálicas de Servicio.

Fuente: Elaboración propia - Unidad Orcopampa.

- Plataforma metálica de trabajo de Pique para colgado de cuadros.

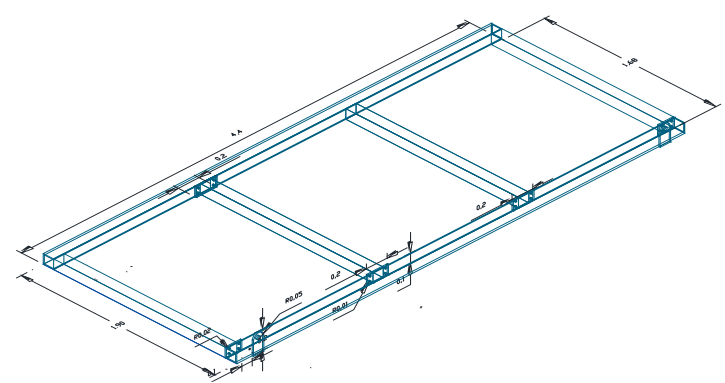

Figura 5:

Isométrico de la plataforma metálica de trabajo.

Fuente: Elaboración propia.

b) Implementación de controles de caída de objetos

- Implementación de controles para evitar caída de objetos: Compuertas de madera en Skip $\mathrm{N}^{\circ} 01$ y $\mathrm{N}^{\circ} 2$.

- Compuertas metálicas eléctricas.

- Implementación de compuertas en los caminos.

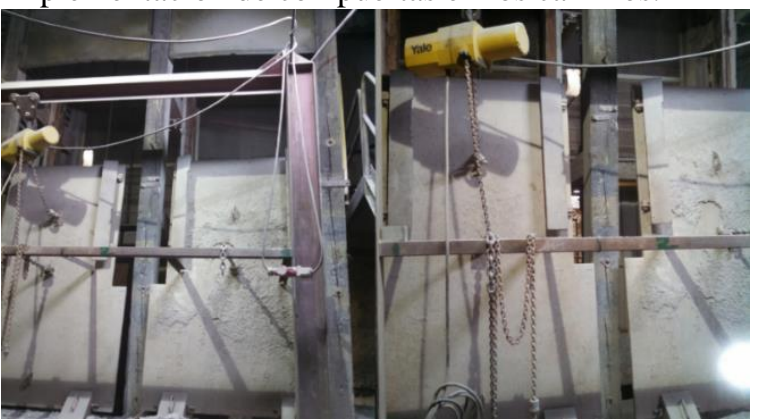

Figura 6:

Compuerta metálica eléctrica.

Fuente: Unidad Orcopampa.

\section{Controles Administrativos}

Este tipo de controles están de acuerdo a la realidad de cada unidad minera, los que se aplicarían en un mismo tipo para todas las minas:

\section{a) Implementación y mejoramiento de la IBD}

Esta herramienta de gestión busca el compromiso y liderazgo por parte de la supervisión en la gestión de la seguridad, el IBD se implementó en la Unidad de Austria Duvaz a partir de junio 2012 y en la Unidad Minera Orcopampa se implementó al inicio de la ejecución del proyecto en el año 2016-2017.

Para realizar el seguimiento y control de los reportes, capacitaciones, elaboración/revisión de PETS, estándares, OPT, etc. la Unidad Orcopampa se implementó un programa informático GRISLI, que permitió mediante los semáforos verificar el seguimiento y cumplimiento de cada una de estas herramientas, estos resultados son expuestos mensualmente en la reunión de gerencias.

Otro punto importante en el análisis de los reportes es la implementación del diagrama de Pareto que permitió determinar el $20 \%$ de las causas que ocasionan el $80 \%$ de accidentes, estas causas son; caída de objetos, condición insegura, desprendimiento de rocas EPP en mal estado, herramientas, incumplimiento a los procedimientos, caída de material, supervisión deficiente, falta de comunicación, mal estado de maquinarias y herramientas, no utilizar EPP, acarreo y transporte $y$ falta de sostenimiento. Este análisis permite tomar las medidas 
ISSNe: 2617-9156

correctivas, implementándose distintos controles a fin de evitar los accidentes en el proyecto de pique.

\section{b) Órdenes de trabajo Mejorado e IPERC Continuo}

Uno de los inconvenientes en el desarrollo del trabajo es el traslape de la información entre la guardia saliente y la entrante. Esta información es de suma importancia, puesto que durante el desarrollo de la guardia se detecta peligros y se aplican los controles respectivos, se recibe recomendaciones de las diversas áreas involucradas como geomecánica, seguridad, ventilación. Al no existir un traslape entre las guardia entrante y saliente se pierde toda información de la secuencia aplicada a los peligros y riesgos, recomendaciones realizadas, acarreando como consecuencia eventos no deseados.

Y uno de los factores para esta deficiencia era la información de las herramientas de gestión como órdenes de trabajo y llenado de IPERC continuo, herramientas en forma individual (hojas separadas), que generalmente debían ser entregadas al final de la guardia y en muchos casos el personal no entregaba. En tal sentido, las recomendaciones y la secuencia de control de los peligros y riesgos no eran informadas al personal de guardia entrante.

Ante esto hecho se implementó en la Unidad de Orcopampa un Cuaderno de Seguridad, el cual permitió transferir toda la información y las recomendaciones realizadas por las distintas áreas en distintas fechas, a fin de continuar aplicando los controles de riesgo respectivo. Así se establecieron las condiciones necesarias de trabajo seguro, empleando el IPER continuo que determinó la naturaleza de la situación adversa.

\section{c) Libro de recorrido del supervisor}

Es un documento de gestión que permite a la supervisión organizarse y priorizar a primera hora las labores de alto riesgo. Esta distribución se realiza en función al informe de la guardia saliente. Se determinan y cubren todas las labores de alto riesgo, priorizando las labores más críticas por toda la supervisión.

Este registro se realiza al inicio de guardia y al culminar se procede a firmar, indicando las observaciones que se encontraron al realizar la inspección y el acto de seguridad realizado. Este libro de recorrido mejoró con la implementación del tipo de roca, en la unidad minera Orcopampa, respecto a la unidad minera Austria Duvaz.

\section{d) Capacitaciones}

Uno de los objetivos de las capacitaciones es brindar y fortalecer conocimientos técnicos y de seguridad, para que los colaboradores desarrollen sus actividades o tareas en forma segura y eficiente. El problema radica en que muchas de estas capacitaciones no son efectivas y aprendidas, lo que se refleja al momento que son abordadas por la supervisión. Al realizar las entrevistas, se evidencia que desconocen o no recuerdan el tema de capacitación que se desarrolló, ya sean externo o interno. En tal sentido, se procedió a mejorar e implementar capacitaciones, entrenamiento, paradas de seguridad, etc. con el fin de elevar el nivel en cultura de seguridad del personal, implementándose las siguientes capacitaciones y entrenamiento:

\section{- Mina Escuela}

Capacitación y entrenamiento teórico - práctico de los colaboradores, se realiza in situ al personal en interior mina. Están en función a los riesgos expuestos por perfil de puestos de trabajo. Se contemplaron temas como: ventilación en minas, control de caída de rocas, sostenimiento y uso de Tabla GSI, perforación y voladura, armado de cuadro colgante, instalación de guías, operación de equipos, operación de herramientas de poder, manipuleo de materiales (izaje de madera), orden y limpieza, las cuales se desarrollaron mediante un programa de Mina Escuela y su seguimiento respectivo. Los encargados de realizar la exposición y entrenamientos son los jefes directos de las áreas de mina y residentes de las empresas contratistas. El seguimiento se realizó mediante informes presentados en la reunión mensual de Gerencia General de la unidad minera.

\section{- Parada de Seguridad}

Actividad que varía de acuerdo a cada unidad minera, en la Unidad Austria Duvaz paralizaba totalmente la producción de una guardia y se desarrollaba con un programa establecido con dinámicas y actuaciones por los colaboradores de cada contratista minera y personal de compañía. Este espacio permitía a los trabajadores dar a conocer los comportamientos inseguros, así como las actitudes negativas por parte de la supervisión, motivando de esta manera al cambio de actitud y realizar los trabajos en forma segura al interior mina.

En la Unidad de Orcopampa se realizó analizando los casos de accidente en las diferentes unidades mineras con el fin de concientizar a los colaboradores en que no se repita la omisión detectada en el análisis.

\section{- PETS y Estándares}

Es de suma importancia mencionar que los PETS, estándares e instructivos son herramientas de gestión que permiten orientar al colaborador la forma correcta de proceder al ejecutar las tareas. Los trabajos en construcción y rehabilitación de piques se realizan por su naturaleza en espacios confinados y en altura, razón por la cual por las características mencionadas este tipo de trabajos se considera dentro de la minería como trabajos de alto riesgo.

\section{Implementación de equipos de poder}

La implementación de equipos de poder está orientada a minimizar el tiempo de exposición del personal el desarrollo de cada una de las actividades y por ende mejorar su rendimiento en el avance y ejecución del proyecto. Dentro de los equipos de poder podemos mencionar:

- Llave de impacto pneumático: 1" 1100 pies / 1 libra: vástago completo.

- Herramienta de poder: Taladro G32 (Taladro 1010W Hp20).

- Uso de winche Canuto.

- Equipo de corte y herramientas de poder para madera (Sierra circular, Cepillo y Disco de Corte (sierra bimetal 
ISSNe: 2617-9156

57 mm (2-1/4") MSS Bosch).

\section{Presentación de resultados \\ Línea base}

Al realizar la línea base se realizó teniendo en cuenta la normativa vigente, Ley 29783 en los siguientes aspectos:

I. Compromiso e Involucramiento. Se tuvo como resultado inicial el $42.5 \%$ de cumplimiento.

II. Política de seguridad y salud ocupacional. Se alcanzó un $47.92 \%$, indica predisposición de la Alta Gerencia por mejorar el sistema de gestión de seguridad y salud en el trabajo considerado malo.

III. Planeamiento y aplicación. Alcanzó una valoración de $26.47 \%$ ubicándolo en el nivel deficiente.

IV. Implementación y operación. Alcanzó una evaluación de $36 \%$ ya que el Comité Paritario no estaba conformado y no estaba implementado el plan de emergencia.

V. Evaluación normativa. Este ítem se encuentra en un $30 \%$, no cuenta con procedimientos para el monitoreo de los cumplimientos del SST, no se cuenta con el reglamento interno de seguridad y salud en el trabajo

Tabla 2.

Cuadro de porcentaje de cumplimientos Según RM 050-2013 TR

\begin{tabular}{llr}
\hline \multicolumn{1}{c}{ Componente } & 2012.04 .05 \\
\hline I. & Compromiso e involucramiento & $42.50 \%$ \\
II. & Política de seguridad y salud ocupacional & $47.92 \%$ \\
III. & Planeamiento y aplicación & $26.47 \%$ \\
IV. & Implementación y operación & $36.00 \%$ \\
V. & Evaluación normativa & $30.00 \%$ \\
VI. & Verificación & $35.42 \%$ \\
VII. & Control de información y documentos & $13.64 \%$ \\
VIII. & Revisión por la dirección & $37.50 \%$ \\
\hline
\end{tabular}

Fuente: Elaboración propia.

En el proceso de implementación e innovación de controles en el proyecto de ejecución de piques, se pudo observar la mejora continua en el proceso de reducción de incidentes incapacitantes y accidentes leves. Se implementaron controles de ingeniería, controles administrativos como PETS y estándares operacionales en las actividades.

En el proceso de Mejora Continua para la Unidad de Austria Duvaz se implementaron 17 controles y se mejoraron 4 controles y en el caso de la Unidad de Orcopampa se
(RISST), no se cuenta con el libro del comité de SST, por todo lo indicado se ubica en un nivel deficiente.

VI. Verificación. Un $35.42 \%$ indica que hay un deficiente análisis de las medidas correctivas del análisis de los accidentes e incidentes peligrosos, se evidencia que no se realizaron auditorías y no cuentan con procedimientos de auditorías.

VII. Control de información y documentos. En el control de información y documentos se tiene el $13.64 \%$, puntaje muy bajo debido a la falta de revisión de los procedimientos, como la falta de recomendaciones de seguridad según puesto de trabajo, no se cuenta con mapa de riesgos.

VIII. Revisión por la dirección. La revisión alcanzó un $37.5 \%$, la cual indica que la alta dirección no revisa periódicamente el sistema de gestión de Seguridad y Salud en el Trabajo.

Todo lo descrito se aprecia en la tabla 2, en la cual el porcentaje de evaluación es de $33.86 \%$ y de acuerdo a la guía RM 050 califica con Nivel Deficiente.

Tabla 3.

Tabla de Resumen de innovación de controles Unidad Austria Duvaz.

\begin{tabular}{lccc}
\hline \multicolumn{4}{c}{ Resumen } \\
\hline Controles & $\mathbf{2 0 1 2}$ & $\mathbf{2 0 1 3}$ & $\mathbf{2 0 1 7}$ \\
Implementados & 7 & 17 & 6 \\
Mejorados & & 4 & 6 \\
\hline
\end{tabular}

Fuente: Elaboración propia.

implementaron 6 controles y se mejoraron 6 controles.

Se puede apreciar en la Tabla 3 que para el año 2013, se implementaron 17 controles y se mejoraron 4 controles en la Unidad de Austria Duvaz, reduciendo los accidentes en un $25 \%$ para fines del año 2012 y en $62.50 \%$ para fines del año 2013.

Por otro lado, en comparación a los indicadores de accidentabilidad de Unidad Austria Duvaz con la unidad de Orcopampa se tuvo una reducción en un $66.67 \%$. 
Tabla 4.

Resumen de Indicadores de Gestión.

\begin{tabular}{|c|c|c|c|c|}
\hline \multirow{2}{*}{ Descripción } & \multicolumn{3}{|c|}{ Austria Duvaz } & \multirow{2}{*}{$\begin{array}{c}\text { Orcopampa } \\
2017\end{array}$} \\
\hline & 2011 & 2012 & 2013 & \\
\hline Accidentes fatales (nivel 5) & 0.0 & 0.0 & 0.0 & 0 \\
\hline Accidentes incapacitantes (nivel 5) & 3.0 & 2.0 & 0.0 & 0 \\
\hline Accidentes triviales (Nivel 2 y 3 ) & 5.0 & 4.0 & 3.0 & 1 \\
\hline Primeros auxilios & 0.0 & 0.0 & 0.0 & 0 \\
\hline Número de trabajadores & 403 & 540 & 589 & 552 \\
\hline Empleados & 96 & 89 & 88 & 72 \\
\hline Obreros & 307 & 451 & 501 & 480 \\
\hline Horas hombre trabajadas & 64554.0 & 109620.0 & 117930.7 & 97328 \\
\hline HHT sin accidentes & 97997.2 & 103072.2 & 146645.7 & 139526 \\
\hline Días perdidos & 49.0 & 44.0 & 10.0 & 0 \\
\hline Índice de Frecuencia & 123.9 & 54.7 & 25.4 & 10.27 \\
\hline Índice de Severidad & 759.1 & 401.4 & 84.8 & 0.0 \\
\hline Índice de Accidentabilidad & 94.1 & 22.0 & 2.2 & 0.0 \\
\hline Accidente de equipos (ADP) & 0.0 & 0.0 & 0.0 & 0 \\
\hline Costos de daños a la propiedad (\$) & 0.0 & 0.0 & 0.0 & 0 \\
\hline Costo total de daños $(\$)$ & 5650.0 & 450.0 & 1100.0 & 500 \\
\hline Cinco puntos de seguridad & 1662.0 & 2208.0 & 2682.0 & 3030 \\
\hline Check list IPERC continuo & 1662.0 & 2208.0 & 2682.0 & 3030 \\
\hline Cuasi accidentes & 0.0 & 0.0 & 0.0 & 0 \\
\hline Condiciones subestándar & 222.0 & 848.0 & 1509.0 & 1964 \\
\hline Actos subestándar & 21.0 & 76.0 & 76.0 & 53 \\
\hline Capacitaciones compromiso (HHC) & 405.0 & 439.0 & 426.0 & 226 \\
\hline Capacitaciones (HH Géminis) & 400.0 & 1770.3 & 2510.0 & 4708 \\
\hline
\end{tabular}

Fuente: Registro propio del investigador.

De acuerdo a la tabla 4, dentro de los indicadores anuales, se tuvo la reducción con respecto a los índices de frecuencia, puesto que para el año 2011 se tiene un $123.9 \%$, para el año 2012 un $54.7 \%$ y cierra el año 2013 con un $25.4 \%$. Con estas experiencias e iniciativas, se realizan las implementaciones y mejora continua a los controles, obteniendo y mejorando en la gestión de seguridad y salud en el trabajo en la Unidad e Orcopampa con un 10.27. En los índices de severidad se redujo de 759.1 en el año 2011 a 401.4 para el año 2012 y 84.8 para el año 2013 , logrando reducir a 0.0 el indicador de severidad en la Unidad de Orcopampa.

En los índices de Accidentabilidad se redujo de 94.1 en el año 2011 a 22.0 para el año 2012 y 2.2 para el año 2013, logrando reducir a 0.0 el indicador de accidentabilidad en la Unidad de Orcopampa.

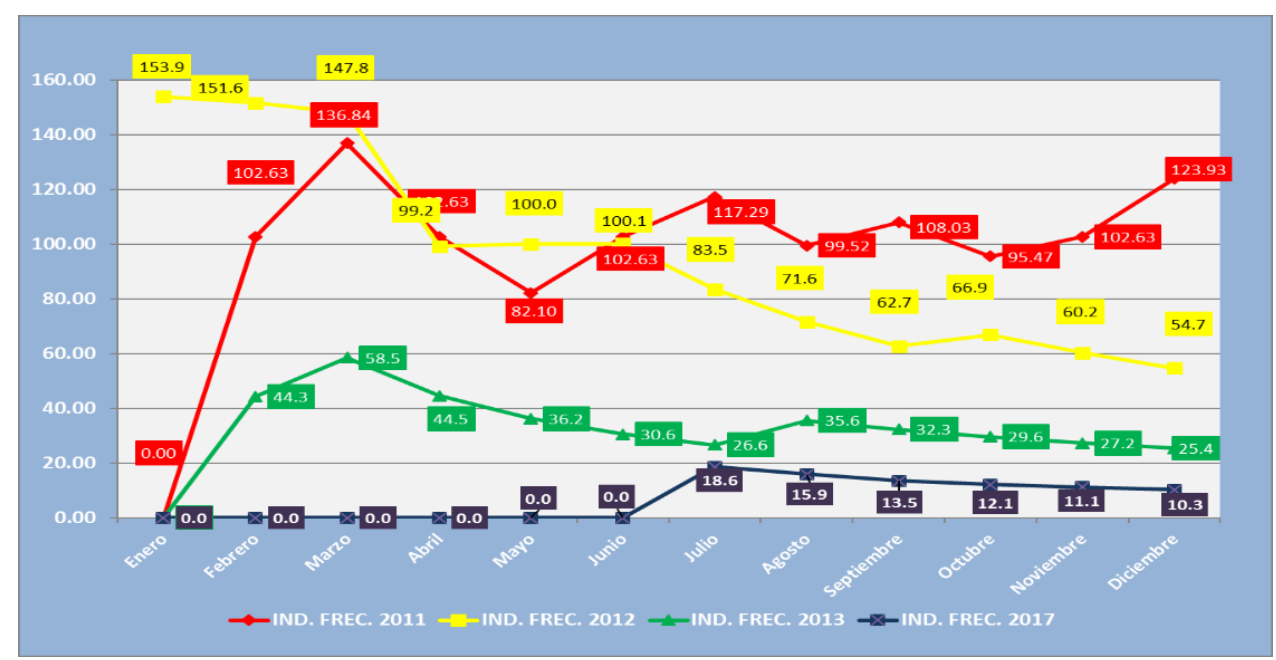

Figura 7:

Índice de Frecuencia

Fuente: Tabla 4. Resumen de indicadores de gestión 


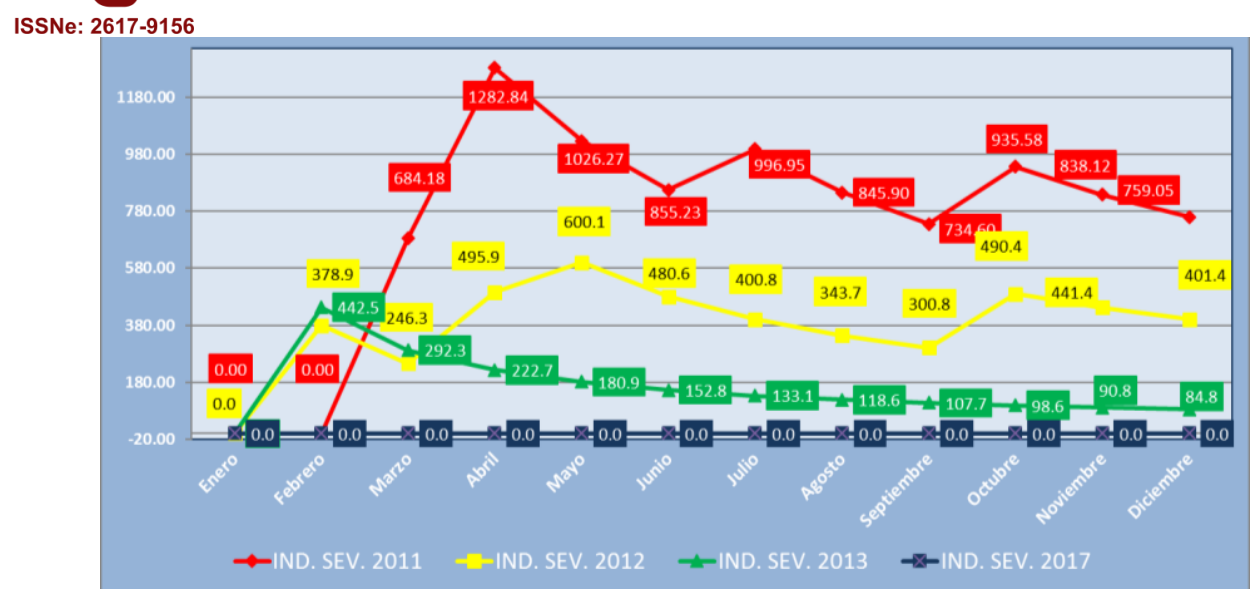

Figura 8:

Índice de Severidad

Fuente: Tabla 4. Resumen de indicadores de gestión.

\section{Figura 9:}

Índice de Accidentabilidad

Fuente: Tabla 4. Resumen de indicadores de gestión.

La evaluación inicial realizada el mes de mayo del 2021 de acuerdo a la tabla 5 alcanzó un $33.68 \%$ ubicando en el nivel deficiente, permitiendo evidenciar los puntos débiles en la gestión del Sistema de Seguridad y Salud en el Trabajo de acuerdo a la Ley 29783, estos datos evidencian la falta de liderazgo, la falta de estándares y PETS en las tareas críticas y las deficiencias en la aplicación de controles a todo nivel.

La Mejora Continúa aplicada a la implementación de controles tecnológicos en el proceso constructivo del pique, se realizó en cada unidad minera, que permitió en forma progresiva la reducción de accidentes y la mejoría de la gestión de riesgos, como se puede apreciar en la tabla 5: de un $33.68 \%$ cierra para el año en un $68.79 \%$ para el año 2013 en la Unidad de Austria Duvaz. Y en un $82.89 \%$ en la Unidad Orcopampa en el año 2017.

Tabla 5.

Evaluación de Línea Base.

\begin{tabular}{rlccc}
\hline \multicolumn{1}{c}{$\begin{array}{c}\text { Evaluación } \\
\text { Capítulo }\end{array}$} & $\mathbf{2 0 1 2}$ & $\begin{array}{c}\text { Resultado } \\
\mathbf{2 0 1 3}\end{array}$ & $\mathbf{2 0 1 8}$ \\
\hline I. & Compromiso e Involucramiento & $42.50 \%$ & $82.50 \%$ & $90.00 \%$ \\
II. & Política de seguridad y salud ocupacional & $47.92 \%$ & $66.67 \%$ & $89.58 \%$ \\
III. & Planeamiento y aplicación & $26.47 \%$ & $55.88 \%$ & $88.24 \%$ \\
IV. & Implementación y operación & $36.00 \%$ & $75.00 \%$ & $80.00 \%$ \\
V. & Evaluación normativa & $30.00 \%$ & $77.50 \%$ & $82.50 \%$ \\
VI. & Verificación & $35.42 \%$ & $79.17 \%$ & $81.25 \%$ \\
VII. & Control de información y documentos & $13.64 \%$ & $63.64 \%$ & $68.18 \%$ \\
VIII. & Revisión por la dirección & $37.50 \%$ & $50.00 \%$ & $83.33 \%$ \\
& & $\mathbf{3 3 . 6 8 \%}$ & $\mathbf{6 8 . 7 9 \%}$ & $\mathbf{8 2 . 8 9 \%}$ \\
\end{tabular}

Fuente: Elaboración propia. 
ISSNe: 2617-9156

\section{Discusión}

A. Viteri (2016) precisa: “... fue importante la evaluación cuantitativa para establecer niveles de riesgo, además de otros controles...”. Entre el 2011 y el 2017 en Austria Duvaz y Orcopampa, siempre se realizaron controles, lo que se aprecia en los resultados es que los controles no solo establecieron los niveles de riesgos, sino los controló y minimizó.

B. Lafaurie y Vargas (2017), indicaron “... la educación del individuo influye en las posibilidades de accidentarse como el hecho más significativo entre otros". La tabla 5, señaló que las capacitaciones fueron incrementándose de 405 a 426 entre 2011 a 2123 en las de Compromiso y las de Géminis de 400 a 2510 en Austria Duvaz y 4708 en Orcopampa, entonces eso refleja en la minimización de los indicadores de frecuencia, accidentes y es favorable para el de seguridad.

C. Canul (2017), que precisaba (parafraseando) "Es importante el análisis documental, para poder favorecer la prevención y el diagnóstico que desarrolló lineamientos, herramientas y controles en una gestión exitosa", de acuerdo a la Tabla 5 el Control de documentos se ha incrementado o mejorado hasta un $68.18 \%$ y lo que muestra que es un factor que permite mejorar las condiciones de seguridad y salud de los trabajadores.

D. Cabello (2018), indicaba "Concluyendo que para minimizar los accidentes por desprendimiento de rocas, es necesaria la participación multidisciplinaria, y una herramienta técnica para logar este objetivo es la guía de criterios geomecánicos que es aporte y resultado de su investigación", el estudio realiza controles técnicos y entre ellos el control geotécnico como se aprecia en las figuras 7 a la 12 que explican sobre el uso de las herramientas y los factores de sostenimiento en el proceso de minado, así como los anexos 1 y 2 que aplica los estudios de resistencia geológica, sumado a la participación de los colaboradores en la mejora de las condiciones de seguridad y salud establecidas.

E. Güere (2018), indicando que “... concluyendo que para minimizar y reducir la probabilidad de los accidentes en el proceso de recuperación de puentes y pilares, es necesario contar con herramientas de gestión óptimos (procedimiento y estándar), y menciona que las minas que vienen trabajando este tipo de actividades por muchos años tendrán menor probabilidad de accidentes que otras que inician estas actividades", dentro del proceso se incluyen los controles administrativos y justamente estos han permitido la minimización de los índices de frecuencia y accidentes, además de mejorar los índices de seguridad, como hechos favorables para ello.

F. Aguilar (2018), señaló “.. el conjunto integrado por los seres humanos y fenómenos, especialmente en la capacitación del enfoque de PHVA IPERC y el nuevo enfoque PHVA IPER que redujo los accidentes". El control administrativo en la correcta aplicación de la matriz en la Tabla 5 donde la evaluación ha permitido apreciar cómo ha ido mejorando en su aplicación y control, lo que ha sido un factor favorable por la reducción de los accidentes.

G. Delzo (2013), indicaba que "la cultura de seguridad tiene relación o asociación con la incidencia de accidentes" en las figuras 29 al 31 se aprecia los procesos de capacitación, instrucción o adiestramiento en la que incluye fortalecer la cultura de seguridad como un hecho relevante para evitar los accidentes, al igual que ellos los procesos aplicados han implicado fortalecer la cultura o conocimientos sobre seguridad como factor relevante de la apreciable reducción de los índices de accidentabilidad. Se puede apreciar que los hechos que se han analizado tienen relación con las investigaciones citadas en la referencia, lo que ha permitido que se corroboren los hechos realizados como favorables en relación con la investigación.

En la tabla 6 se puede observar la innovación en forma continua de los controles de herramientas de la gestión de seguridad como: IPERC Continuo, PETS, Estándares, instructivos, RIACS, que se realizó a partir del año 2011 en la mina Austria Duvaz hasta el año 2017 en la Unidad Orcopampa.

Tabla 6:

Herramientas de Gestión de Seguridad, implementados e innovados.

\begin{tabular}{cccccc}
\hline Control & $\begin{array}{c}\text { IPERC } \\
\text { continuo }\end{array}$ & PETS & Estándares & Instructivos & RIACS \\
\hline $\mathbf{2 0 1 1}$ & 1662 & 8 & 3 & 0 & 243 \\
$\mathbf{2 0 1 2}$ & 2208 & 12 & 7 & 2 & 924 \\
$\mathbf{2 0 1 3}$ & 2682 & 48 & 13 & 4 & 1585 \\
$\mathbf{2 0 1 7}$ & 3030 & 61 & 33 & 5 & 2190 \\
\hline
\end{tabular}

Fuente: Elaboración propia.

\section{Conclusiones}

- Los indicadores de gestión de seguridad reflejan su influencia positiva en la aplicación de los controles a partir del año 2012, pues el Índice de Frecuencia con respecto al año 2011 para el año 2012 se redujo en un $55.83 \%$, para el 2013 se redujo en un $-79.47 \%$ en la Unidad Austria Duvaz y para el año 2017 se redujo en un Attribution-NonCommercial 4.0 International (CC BY-NC 4.0)
$-91.71 \%$ en la Unidad Orcopampa. El índice de Severidad se redujo en un $-47.12 \%$ para el 2012 , en un $-88.83 \%$ para el 2013 y en un $100 \%$ para el año 2017

- En la evaluación del sistema de gestión de seguridad a partir de la línea base se puede apreciar el compromiso por parte de la alta gerencia, el liderazgo asumido por la supervisión y de los colaboradores, pasando de un nivel deficiente en el mes de mayo 2012 a un nivel regular para el año 2013 en la Unidad Austria Duvaz y nivel bueno en 
ISSNe: 2617-9156

el año 2017 en la Unidad Orcopampa.

- En la presente investigación se determinó el efecto positivo de la implementación de los controles tecnológicos en la reducción de accidentes en la construcción de piques de la Corporación Minera Géminis SAC, pues a partir del año 2011 al año 2012 se redujo en un $25 \%$ y para el año 2013 se redujo en un $62.50 \%$ en la Unidad Austria Duvaz, por otro lado, en el proceso de mejora continua se redujo en un $66,67 \%$ en la Unidad Orcopampa.

- En la presente tesis se determinó que la implementación y la innovación de equipos de poder y equipos influyen en la reducción de accidentes en la construcción de piques de la CM Géminis SAC

- Se determinó que es de suma importancia el monitoreo y la implementación de los programas geomecánicos en la reducción de accidentes en la construcción de piques de la CM Géminis SAC.

\section{RECOMENDACIONES}

- Mejorar el desarrollo de las auditorías internas y externas al sistema de gestión de seguridad a fin de detectar oportunamente las desviaciones en la gestión de seguridad y a partir de estas, realizar los planes de acción para las desviaciones.

- Generar una cultura de innovación e implementación de controles tecnológicos para una mejora continua de la gestión de seguridad en los proyectos de ejecución de piques.

- Fortalecer la implementación de los diversos programas geotécnicos para realizar un seguimiento del comportamiento estructural del macizo rocoso y así prevenir oportunamente los eventos no deseados.

- Fortalecer el uso de las herramientas de gestión de seguridad como las órdenes de trabajo, IPERC continuo, OPT, ATS, PETAR y realizar un monitoreo mediante un

- software a fin de evaluar la eficacia de éstas.

\section{REFERENCIAS}

[1] Angeles, C. H. y Cueva, R. J. (2019). Optimización en la planificación minera a tajo abierto (Tesis de Pregrado). Universidad Privada del Norte, Cajamarca.

[2] Baldera, J., Medina, J. y Uriarte, L. (2017). Evaluación del impacto de la accidentabilidad en la productividad de las minas peruanas. Casos de estudio: empresas de la BVL en la gran y mediana minería (Tesis de Maestría). Escuela de Postgrado Gerens, Lima.

[3] Ballido, J. y Giraldo, M. (2015). Technical and economics consequences of fatal accidents in peruvian mining. Instituto de Investigación (RIIGEO). 18, 11.

[4] Bavaresco, G. (2009). Historia de la seguridad indutrial y prevención de accidentes. Puerto Cabello, Venezuela: GABP.

[5] Cooperativa.cl. (11 de febrero de 2018). Accidentes Laborales. Santiago de Chile. Obtenido de https://www.cooperativa.cl/noticias/pais/trabajo/accide ntes-laborales/trabajador-murio-tras-accidente-endivision-andina-de-codelco/2018-02-11/114721.html

[6] De la Cruz, E. (2000). Seguridad en el manejo y operación de piques. Instituto de Investigación (RIIGEO), 3(05), 20-25.

[7] Delzo, A. (2013). Influencia de la cultura de seguridad en la incidencia de accidentes con maquinarias pesada en las concesiones mineras de la región Junin. (Tesis de Maestría). Universidad Nacional del Centro del Perú, Huancayo.

[8] Eurostat (2018). The Measurement of Scientific, Technological and Innovation Activities - Oslo Manual 2018 - Guidelines

[9] Fernández, R. (2016). Optimización del proceso de revestimiento con concreto mediante uso del sistema slick line para el transporte de concreto en la construcción del pique principal Marza. (Tesis de Pregrado). Universidad Nacional de Trujillo, Trujillo.

[10] Giraldo, M. y Badillo, J. (18 de Mayo de 2015). Implicancias técnicas y económicas de los accidentes mortales en la mineria peruana. Revista del Instituto de Investigación (RIIGEO), FIGMMG-UNMSM, 18(35), 97-107.

[11] Herrera, V. (2013). La escasa implementación de tecnologias en cooperativas mineras en el municipio de Tipuani. (Tesis Pregrado). Universidad Mayor de San Andrés, La Paz.

[12] Furtado de Oliveira, P. (26 de 4 de 2019). Conferencia sobre Seguridad y Salud de trabajadores adolescentes y jóvenes. Obtenido de Infobae: https://www.infobae.com/america/mundo/2019/04/26/1 a-oit-revelo-que-7-600-personas-mueren-por-dia-en-elmundo-como-consecuencia-de-accidentes-de-trabajoo-enfermedades-profesionales/

[13] López, J. y Pérez, G. (2013). Propuesta metodológica para el mejoramiento del proceso de piking con base en el enfoque de Harrington y el estudio de métodos de la OIT. Saber, ciencia y libertad, 8 (2), 93-113.

[14] Montero, R. (19 de Abril de 2018). Aporte de la minería al PBI. Obtenido de El Peruano: https://elperuano.pe/noticia-aporte-de-mineria-al-pbi65726.aspx

[15] Oficina Estadistica MINTRA. (2018). Anuario 
ISSNe: 2617-9156

Estadístico Sectorial Ministerio de Trabajo y Promoción del Empleo. Lima.

[16] Salinas, C. (1998). Construcción de Tuneles, Piques y Chimeneas. Santiago de Chile: Departamento de Ingenieria de Minas de la Universidad de Chile.

[17] Soto, I. (s.f.). Cultura de Seguridad. Prevencionar, México. Obtenido de: https://prevencionar.com/2011/09/15/cultura-de-laseguridad/

[18] Suárez-Barraza, M. F. y Miguel-Dávila, J. A. (2009). Encontrando al Kaizen: Un análisis teórico de la mejora continua. Revista de la Facultad de Ciencias Económicas y Empresariales-Universidad de León, México, 2008 (7), 285-311.

[19] Vallejo, L. (2015). Características de los programas de capacitación andragógicos y los procesos de aprendizaje en la seguridad minera peruana (Tesis de Maestria). Universidad Nacional Mayor de San Marcos, Lima. 